Aus der I. Medizinischen Abteilung des Allgemeinen

Krankenhauses Hamburg-Barmbeck.

(Direktor: Prof. Th. Rumpel.)

\title{
Zur Frage der Relaxatio (Eventratio) diaphragmatica.")
}

Von Dr. Jacques Neumann, Sekundärarzt.

Die von Cruveilhier geprägte Krankheitsbezeichnung der Eventratio diaphragmatica ist irreführend, da man sich dabei unwillkürlich die Verlagerung von Eingeweiden aus der Bauchhöhle in die Brusthöhle durch einen Spalt im Zwerchfell hindurch vorstellt, wie es in Wirklichkeit nur bei der Zwerchfellhernie der Fall ist. Bei der Eventratio dagegen ist die Kontinuität des Diaphragmas mit seinem Pleura- und Peritonealüberzug vollkommen gewahrt, das erschlaffte Zwerchfell ragt sackförmig weit in die Brusthöhle hinein, während sich in die unterhalb gelegene Kuppelwölbung Abdominalorgane einlagern.

Zur Ausmerzung dieser unglücklich gewăhlten Benennung sind mehrere Vorschlăge gemacht worden. Königers idiopathischer, einseitiger Zwerchfellhochstand ist zu nichtssagend und in der Idiopathie nicht ohne weiteres gültig, wie in den folgenden Ausführungen ersichtlich sein wird. Franks Zwerchfellinsuffizienz trifft das Charakteristische des Krankheitszustandes zu wenig, während die Relaxatio nach Wieting das Wesentliche der Affektion zwar nicht völlig erschöpft, ihr aber doch am nächsten kommt. Alle neueren Autoren haben daher diese Bezeichnung akzeptiert.

Die Relaxatio diaphragmatica (R. d.) galt früher als eine ganz außerordentlich seltene Erscheinung. Bis zum Jahre 1899 konnte Grosser ${ }^{1}$ ) nur acht Fälle aus der Literatur zusammenstellen, die ohne Ausnahme als Zufallsbefunde bei Autopsien festgestellt waren. Der zunehmenden Vervollkommnung des Röntgenverfahrens danken wir nun einmal eine stattliche Vermehrung der Beobachtungen und dann den Fortschritt, da $\beta$ die R. d. bereits am Lebenden erkannt werden konnte. Ich möchte allerdings schon hier darauf hinweisen, daß röntgenologisch zurzeit noch eine sichere Differenzierung der $R$. d. von der weit häufigeren Hernia diaphragmatica $(1: 50)$ auf ungeahnt große und manchmal unüberwindliche Hindernisse stößt und daher die Sicherheit der Diagnose in all den beschriebenen Fällen, soweit sie nicht zur Sektion gelangten, keine apodiktische genannt werden kann.

1913 stellte Bergmann ${ }^{2}$ ) bereits 22 sichere und 5 wahrscheinliche Relaxationen fest, wozu noch die 1912 von Lorey ${ }^{3}$ ) veröffentlichten 5 Fălle kommen, von denen 2 sogar durch die Sektion ihre Bestätigung fanden. 1916 demonstrierte Weil ${ }^{4}$ ) den Röntgenbefund von 5 Patienten mit R. d. Es ist interessant und für die Praxis wichtig, unter welchen Diagnosen diese letzteren Fälle zur röntgenologischen Untersuchung kamen. Ich will sie daher in Kürze anführen: beim 1. war die Verdrăngung des Herzens nach rechts für eine Herzerweiterung gehalten worden, beim 2. und 3. war eine Pleuritis, beim 4. eine zweifelhafte Lungenerkrankung und beim letzten eine Erkrankung des Magendarmkanals angenommen worden.

In neuester Zeit vermehrte sich die Zahl der klinischen Beobachtungen noch um drei, und zwar je eine von Roesch ${ }^{5}$ ), Andree ) und Assmann $n^{7}$. Wir haben also bei Zurücksetzung der oben angeführten Bedenken gegen die Sicherheit der röntgenologischen Diagnose bis zum Jahre 1919 inklusive der wahrscheinlichen Fälle 41 Relaxationen, von denen 19 durch die Autopsie sichergestellt worden sind.

Wir sind in der Lage, einen weiteren klinisch diagnostizierten Fall mitteilen zu können dessen eindeutiger anatomischer Befund uns neben anderen Beobachtungen Gelegenheit gibt, zur Frage der Pathogenese der R. d. Stellung zu nehmen.

Ich lasse zunächst die Krankengeschichte folgen:

Der am 2. März 1917 aufgenommene, 44jährige Major $R$. gab zur Anamnese an, früher nie ernstlich krank gewesen zu

*) Eingegangen am 21. III. 19.

1) W. kl. W. 1899 Nr. 24. - 2) Ergebn. d. Inn. Med. u. Kindhlk. 12 (hier auch ausführlich die frühere Literatur). - 8) Verh. d. deutschen Röntgengesel lsch. 8. - 4) Vereinig. $d$. kriegsärztl. beschäftigten Aerzte, StraBburg 25. I. 16. Ref. D. m. W. 1916 S. 744. - 5) Inaug. Diss. Breslau 1918. - ') M. Kl. 1918 Nr. 40. - ') Fortschr. d. Rönt genstr. 26 H. ${ }^{r} 1$. 
sein und seit seinem 19. Lebensjahre ununterbrochen im militärischen Dienste gestanden zu haben. Während des Krieges war er zweimal - 1914 und 1915 - wegen eines nervösen Erschöpfungszustandes längere Zeit in Lazaretten bzw. Kurorten in Behandlung gewesen. Im Januar 1917 habe er etwas Husten bekommen und sei deshalb Anfangs Februar zum Arzte gegangen, der einen leichten Bronchialkatarrh festgestellt habe. Am Vormittage des Aufnahmetages erkrankte er plötzlich mit Schüttelfrost, geringem Husten ohne Auswurf, Stichen in der rechten Brustseite, Mattigkeits- und Schlappheitsgefühl. Auf nachträgliches, genaueres Befragen gab er noch an, früher niemals Atem-, Herz- oder Magenbeschwerden gehabt zu haben, dagegen immer, $d . h$. solange er sich zurück erinnern kann, in der linken unteren Brustseite das Gefühl gehabt zu haben, als ob dort ,etwas nicht in Ordnung sei", doch habe er diesen geringfügigen Sensationen keinerlei Bedeutung beigelegt.

Bei der Aufnahme bot der Patient folgendes Bild: Mittelgroßer, leidlich kräftiger Mann in entsprechendem Ernährungszustande. Sprechen mühsam, häufig von trockenem Hustenreiz unterbrochen. Atmung oberflächlich, sehr beschleunigt. Geringe Zyanose der Lippen. Im ganzen etwas unruhig, aber bei vollem Bewußtsein.

Thorax: völlig symmetrisch, die linke Seite bleibt beim Atmen etwas zurück.

Lungen: Im Bereiche des rechten Unterlappens tympanitische Dämpfung, bronchiales Atmen, klein- und mittelblasiges, feuchtes Rasseln und verstärkter Stimmfremitus. Links hinten unten von der Mitte der Skapula an nach abwärts besteht eine Dämpfung von mäBiger Intensität und leicht tympanitischem Beiklange. In ihrem Bereiche ist das Atemgeräusch aufgehoben, ebenso der Stimmfremitus; man hört anscheinend fortgeleitetes leises Rasseln und hier und da vereizelte eigenartig schlürfende und glucksende Geräusche. Sputum gering, rostfarben

Herz: In der Gegend der normalen Herzdämpfung findet sich tympanitischer Klopfschall. Diese Tympanie reicht nach oben bis zur dritten Rippe, von da an heller Lungenschall mit reinem Vesikuläratmen, während im Bereiche der Tympanie ganz leises, fernklingendes Atmen vernehmbar ist. Die Herzdämpfung reicht rechts bis einen Querfinger breit innerhalb der rechten Mamillarlinie, wahrend die linke Grenze am linken Sternalrand liegt. Der Spitzenstoß ist nicht sicht- und fühlbar. Die Herztöne sind im Bereiche der linkseitigen Tympanie ganz leise (fortgeleitet) zu hören. Ueber der rechtseitigen Herzdämpfung sind sie rein und kräftig, an der Basis Verstärkung des zweiten, dem Brustbein näherliegenden Tones (Pulmonalis!). Aktion regelmäßig, leicht beschleunigt, 104 Schläge in der Minute bei einer Temperatur von $38^{\circ}$. Der Puls ist gut gefüllt, kräftig.

Abdomen: Weich, keinerlei Druckempfindlichkeit. Leber nicht vergrößert, keine nachweisbare Milzschwellung.

Im übrigen kein weiterer pathologischer Befund

Wir nahmen nach diesem Befunde eine kruppöse Pneumonie des rechten Unterlappens an. Ueber die Dämpfung links hinten unten und über die Ursache der Dextrokardie waren wir uns anfänglich ${ }_{i}$ nicht ganz im klaren; wir vermuteten, daß es sich um eine ausgedehnte Pleuraschwarte handle, die durch Schrumpfung das $Z$ werchfell außen und oben fixiert und dadurch ein Abgleiten des Herzens nach rechts herbeigeführt habe.

Das Fieber dauerte in den nächsten Tagen ohne Veränderung der pneumonischen Erscheinungen kontinuierlich um $39^{\circ}$ an. Subjektiv bestanden außer Husten und rechtseitigen Bruststichen keine Beschwerden, kein Gefühl der Kurzatmigkeit. Im Gegensatze dazu fiel uns eine zunehmende Zyanose auf, die sich besonders an den Fingerspitzen und an den Ohren bemerkbar machte. Am 7. März trat auch im rechten Oberlappen Dämpfung mit bronchialem Atmen auf, während im Unterlappen zahlreiches mittelblasiges Rasseln hörbar war. Wir hatten bisher vom ersten Krankheitstage an Optochin gegeben, setzten dasselbe aber ab, da wir keinerlei subjektive und objektive Einwirkung auf die Erkrankung sahen. Im weiteren Verlaufe sank die Temperatur etwas ab und nahm einen remittierenden Charakter an. Die Dämpfung über beiden Lungenlappen rechts nahm an Intensität $\mathrm{ab}$, das Bronchialatmen und die katarrhalischen Erscheinungen blieben aber unverändert bestehen. Der Puls war bisher kräftig und ruhig gewesen, seit dem 15. März aber trat eine erhebliche Tachykardie auf. Das Auffallendste war die zunehmende Zyanose, deren Stärke uns bei den fehlenden ausgesprochenen Herz:insuffizienzerscheinungen durch die Lungenentzündung allein nicht genügend erklärt schien, sodaß wir an Tuberkulose, eventuell an eine miliare Aussaat dachten.

Um uns darüber und über die linkseitige Dämpfung und Dextrokardie Klarheit zu verschaffen, wurde am 23. Marz eine Röntgenuntersuchung (Prof. Dr. Haenisch) vorgenommen. Dalsei fanden sich im rechten Ober- und Unterlappen diffuse fleckige Abschattungen und ein rechtseitig lateral adhärentes Zwerchfell. Im linken Lungenfeld ist eine feine Schwarte sichtbar. Der Hilus ist beiderseits verdichtet. Links spannte sich in Höhe der vierten Rippe bogenförmig ein schmaler, bandartiger Schatten vom Mittelfelde zur Thoraxwand aus. Bei der Durchleuchtung zeigte das Zwerchfell rechterseits leidlich gute Exkursionen, wahrend der beschriebene, bogenförmige Schatten nur ganz geringe, aber dem rechten gleichsinnige, nicht paradoxe Bewegungen aufwies. Unterhalb des bandförmigen Schattens war die Magenblase und die Magenwand gut erkennbar. Lateral davon, teilweise hinter ihr, sind gashaltige Dickdarmschlingen durch die Haustrenbildung gut differenzierbar. Das Flüssigkeitsniveau des Magens liegt noch zwei Querfinger oberhalb der rechten Zwerchfellkuppe. Die Magenblase selbst ist etwa $2^{1 / 2}$ Querfinger hoch. Auf mehreren Platten zeigte sich die Unveränderlichkeit der Grenzbogengestaltung. Die Herzsilhouette ist stark nach rechts verlagert.

Von weiteren feineren röntgenologischen Untersuchungen mußte des schlechten Befindens des Patienten wegen leider abgesehen werden.

Röntgenologische Diagnose: Pneumonische Infiltrationen im rechten Lungenfeld. Linkseitige Eventratio diaphragmatica. Dextrokardie. Weiterer Krankheitsverlauf: Die Temperatur blieb weiterhin unter $37^{\circ}$, während der Puls, zwischen 100 und 120 schwankend, weich und klein wurde. Blutdruck (Riva-Rocci) 98. Die Lungenerscheinungen änderten sich nicht; es bestand ständig geringer Husten und wenig zäher, schleimigeitriger Auswurf ohne Tuberkelbazillen.

Die Zyanose nahm weiterhin zu. Es traten dann häufig kollapsartige Anfälle mit Schweißausbruch und erheblicher Dyspnoe ein, später zunehmender Verfall. Am 11. April 1917 starb der Patient.

Die klinische Diagnose lautete: Unvollkommen gelöste Bronchopneumonie im rechten Ober- und Unterlappen. Linkseitige R. d. mit Dextrokardie.

Bei der Sektion (Prof. Dr. Fahr) stand die linke Zwerchfellkuppe auffallend hoch, am unteren Rande der dritten Rippe, während das rèchte Zwerchfell bis zum unteren Rande der fünften Rippe reichte. Unterhalb der linken Zwerchfellkuppe liegt der stark nach oben verlagerte Magen und die linke Flexura coli, während die Milz ihre normale Lage nicht verändert hatte.

Nach Eröffnung der Brusthöhle sank die linke Zwerchfellhälfte völlig schlaff in sich zusammen. Die Muskulatur des linken Zwerchfells war so dün und atrophisch, daß es völlig durchsichtig war und einem dünnhäutigen Sacke ähnelte. Zwischen dem Hilus der linken Lunge und dem äußeren Herzbeutelblatte fanden sich schwielige Verwachsungen. Das äußere Blatt des Herzbeutels ist an dieser Stelle sehr verdickt und weißlich verfärbt. Der N. phrenicus zieht durch diese schwielig verănderte Partie und ist hier auffallend verdannt, nicht dicker als ein Zwirnsfaden. Auch in den übrigen Partien ist er erheblich dünner als auf der anderen Seite.

Das Herz ist stark nach rechts verlagert. Die Herzklappen sind, abgesehen von flachen Verdickungen an der Mitralis, intakt. Die Muskulatur des rechten Ventrikels ist deutlich verdickt. An der Innenfläche der Koronarien finden sich flache, gelbliche Intimaverdickungen, desgleichen an der Innenfläche der Aorta. Herzgewicht $310 \mathrm{~g}$,

Die Schleimhaut der Bronchien ist geschwollen und gerötet. In den unteren Abschnitten des rechten Unter- und Oberlappens finden sich größere, luftleer infiltrierte Bezirke von schlaff elastischer Konsistenz und schwärzlicher Schnittfläche. In der linken Lunge keine Kompressionserscheinungen.

Gesamtbefund: Atrophie des linken N. phrenicus und der linken Zwerchfellhälfte. Pleuritische Verwachsungen zwischen dem linken Lungenhilus und äußerem Blatte des Herzbeutels. Bronchopneumonische Herde. Bronchitis. Hypertrophie des rechten Ventrikels. Koronarsklerose. Arteriosklerose.

Die mikroskopische Untersuchung des linken Zwerchfells zeigte nur spärliche, verschmälerte und kernarme Muskelfasern, zwischen denen das Fettgewebe stark gewuchert war: Muskeldegeneration mit sekundärer Fettinfiltration. Die mikroskopischen Veränderungen am linken Phrenikus traten besonders deutlich beim Vergleiche mit dem rechten Nerven nach van Gieson-Färbung zutage. Der gesunde Nerv ist zusammengesetzt aus weißlichgelblichen Nervenfasern, umgeben von rot gefärbtem Bindegewebe, der kranke, sehr dünne Nerv zeigt schwielige Atrophie, besteht nur aus Bindegewebe ohne jede Nervenfaser.

Die geschilderten makroskopischen Veränderungen am Zwerchfell - Hochstand, Schlaffheit und häutige Verdünnung sind nach den vorliegenden übereinstimmenden Literaturangaben als typisch für die R. d. zu bezeichnen. Mikroskopische Beobachtungen liegen bisher nur in drei Fällen vor. Benda fand an dem von Glaser ${ }^{1}$ ) publizierten Falle an Stelle der makroskopisch scheinbar vorhandenen Muskelfasern parallele Stränge von runden Fettzellen, die ungefähr die Breite von Muskelfasern eingenommen hatten. $\mathrm{Er}$ sieht deshalb in diesen Veränderungen des atrophischen Zwerchfells eine Art lipomatöser Pseudohypertrophie, wie sie sich bei der myogenen Muskelatrophie findet.

Etwas anders waren die Veränderungen, die Motzfeld ${ }^{2}$ ) sah. Er fand überwiegend Bindegewebe in Gestalt von zellarmen, stark fibrinösen Zügen, zwischen denen spärliche, schmale Streifen Fettgewebe lagen, oft nur als eine einzelne Reihe von Fettzellen, wăhrend er von Muskelelementen anscheinend nichts mehr vorfand. Döring ${ }^{3}$ ) beschreibt an den untersuchten Stellen des

1) D. Arch. f. kl. Med. 78. 1903. - ') D. m. W. 1913 Nr. 7. - 
Zwerchfells nach der Kuppe der Ausbuchtung hin zunehmende Verminderung der Muskelfasern, die im Verhältnis der Dichte des sie einschließenden Bindegewebes in mehr oder weniger erheblichem Grade Druckatrophie zeigten.

Wir sehen also, daß die degenerativen Veränderungen am Zwerchfell keine einheitlichen sind; auf der einen Seite (Motzfeld und Döring) bindegewebige, auf der anderen Seite (Benda und wir) fettige Degeneration. Die Unterschiede zwischen unserem und dem Bendaschen Befunde können nur als graduelle aufgefaßt werdeh, in dem Sinne, da $B$ in unserem Falle ein früheres Stadium der Bendaschen Pseudohypertrophie vorzuliegen scheint.

Auf die bisher gefundenen Veränderungen am Phrenikus werde ich bei der Pathogenese der Relaxatio zu sprechen kommen. Zunächst will ich mich der Klinik der R. d. zuwenden.

Wir haben bei unserem Falle gesehen, da $\beta$ die $R$. d. ohne Beschwerden bei ihrem Träger bestand. Diese Symptomlosigkeit dürfte die Regel sein. Selbst wenn subjektive Erscheinungen vorhanden sind, sind sie zumeist nur sehr gering und so uncharakteristisch, daß die Anamnese keineswegs den differentialdiagnostischen Spürsinn auf die Erkennung einer R. d. hinlenken wird. Von seiten des Verdauungskanals wird zumeist nur leichtes Druck- oder Völlegefühl in der linken Seite geăußert, und nur Glaser ${ }^{1}$ ) beobachtete bei seinem Patienten tagelanges Erbrechen und öfters Hämatemesis ohne Ulzeration, während sich in einem Falle von $\mathrm{Herz}^{2}$ ) das Bluterbrechen durch ein gleichzeitig bestehendes Magengeschwür mit Sanduhrbildung erklärte. Allerdings bringt Herz das Geschwür in ursächliche Beziehung $\mathrm{zu}$ der durch die Relaxatio bedingten Dislokation des Magens.

Trotz der hochgradigen Veränderungen der Raumverhältnisse im Thorax sind Klagen übre Lungen- oder Herzbeschwerden äußerst selten. Dyspnoe, Zyanose und Herzklopfen werden fast stets vermißt, nur leichtes Ziehen zwischen den Schulterblättern oder geringe stechende Schmerzen in der unteren Brustseite werden in der Regel von den Patienten angegeben.

Was die klinische Erkennung der R. d. betrifft, so sind es drei Kardinalpunkte, die den Verdacht auf ihr Vorhandensein lenken müßten. Ich führe die linkseitige R. d. als typisch an, weil die überwiegende Mehrzahl des Leidens auf dieser Seite gefunden wird. Diese drei Kardinalsymptome sind:

1. eine mehr oder - weniger ausgesprochene Dextrokardie, 2. eine

3. eine Dämpfung links hinten unten, deren Intensität wechseln kann, in der das Atemgeräusch aufgehoben ist und in deren Bereich man eventuell hier und da eigentlimliche plätschernde oder glucksende Geräusche hören kann.

Trotzdem nun diese Symptome in ausgesprochener Weise und mit Sicherheit schon sehr lange bei unserem Patienten vorhanden waren und trotzdem er sich während seiner langen Dienstzeit sehr häufigen ärztlichen Untersuchungen unterziehen mußte, wurde die R. d. nicht erkannt. Noch in den Krankengeschichten der Lazarette, in denen sich der Patient 1914 und 1915 befand, ist in bezug auf Lunge und Kreislaufsystem in der einen nur von einer Pulsbeschleunigung, in der anderen nur von einer Verbreiterung des Herzens um zwei Querfinger breit nach rechts die Rede. Ich führe diese Tatsachen an, um zu zeigen, daß die $R$. d. sicher häufig übersehen wird und daß sie ohne Zweifel weit häufiger vorkommt, als sie entdeckt, wird.

Die klinische Diagnose ist insbesondere deswegen so selten, weil das Krankheitsbild noch nicht genügend allgemein bekannt ist und infolgedessen an die Möglichkeit seines Vorhandenseins nicht gedacht wird. Die meisten beobachteten Fälle wurden erst durch das Röntgenverfahren aufgedeckt. Es ist deshalb dem Röntgenologen die R. d. ein geläufiger Begriff, der sich in allen modernen einschlägigen Büchern findet, während in den medizinischen Werken bisher nur Eppinger ${ }^{3}$ ) diese Affektion erwähnt. Auch auf chirurgischer Seite hat bislang die $\mathrm{R} d$. ein größeres Interesse für sich gehabt, da sie in differentialdiagnostischer Konkurrenz zu der chirurgisch bedeutsamen Zwerchfellhernie tritt.

Bei den differentiell in Frage kommenden Tumoren und Abszessen der Lunge oder Pneumothorax ist der Entscheid hauptsächlich mittels der Radiologie nicht schwer. Anders dagegen bei der Zwerchfellhernie. So völlig klar die anatomischen Bilder der R. d. und der Zwerchfellhernie zu scheiden sind, so schwierig ist ihre klinische Differenzierung. Die Hernie ist ja an und für sich die weitaus häufigere Krankheit - nach der Zusammenstellung von Grosser kommen auf $10 \mathrm{R}$. d. 500 Zwerchfellhernien - und es wird in der Regel ein Leichtes sein, eine Hernie zu erkennen, wenn starke Beschwerden (Zyanose, Herzklopfen, Schmerzgefühl in der Brust nach dem Essen) oder gar Einklemmungserscheinungen vorhanden sind. Ich möchte hier kurz einen einschlägigen Fall erwähnen, den wir in letzter Zeit beobachtet haben. Es hạndelt sich um einen fünfjährigen Jungen, der wegen periodischen Erbrechens mit Azetonurie bei uns lag, bei dem die Sektion eine linkseitige Zwerchfellhernie aufdeckte. Der in die Brusthöhle verlagerte Magen war mit der Lunge und dem Zwerchfell erheblich verwachsen, wodurch wohl das dauernde Erbrechen

1) l. c. - 2) W. kl. W. 1907 Nr. 47. - ') Suppl. I zu Nothnagels pez. Pathol. u. Ther. seine Erklärung findet. Eine Röntgenuntersuchung hatte leider nicht stattgefunden. Ich erwähne diesen Fall, um zu zeigen, daß derartige stärkere Beschwerden im Zweifelsfalle natürlich eher für eine Hernie sprechen dürften als für eine R. d.

$\mathrm{Da}$ ferner nach einer statistischen Zusammenstellung von Grosser jede zweite Hernie durch Unfall erworben ist, so könnte man ein Trauma in der Anamnese für die Hernie verwerten. Dazu ist aber zu bemerken, daß einerseits eine $R$. d. auf dem Umwege uber eine traumatische Phrenikusverletzung entstehen kann - ich komme auf diese Möglichkeit bei der ätiologischen Erörterung ausführlich zurück - anderseits ein Zusammenhang zwischen R. d. und Trauma in dem Sinne möglich ist, daß eine subjektiv symptomlos verlaufende $R$. d. durch die Einwirkung von äußerer Gewalt zu Beschwerden führen und dadurch zur Entdeckung gebracht werden kann.

Weit größer und klinisch kaum überwindbar werden die Schwierigkeiten in der Differentialdiagnose dann sein, wenn wie es zumeist der Fall ist, auch die Hernie ebenso wie die R. d. ohne wesentliche Beschwerden besteht, da die obengenannten klinischen Hauptsymptome der R. d. genau in der gleichen Weise auch bei der Hernie vorhanden sein können.

Alle Versuche, auf rein klinischem Wege die differentialdiagnostische Entscheidung herbeizuführen, sind bislang als gescheitert zu betrachten. Druckmessung mittels in den Magen eingeführten Manometers (Struppler), Feststellung der verminderten Vitalkapazität der erkrankten Seite (Struppler, Baetge) haben sich ebensowenig eindeutig und zuverlassig erwiesen als die Gástrodiaphanie (Hildebrandt-Hess).

Dagegen versprachen die Röntgenstrahlen eine einfache und mühelose Klärung der Frage. Aber auch hierin sah man sich getäuscht, weil bei den gewöhnlichen Untersuchungen die röntgenologischen Bilder für beide Affektionen die gleichen sind. Man findet nämlich sowohl bei der R. d. wie bei der Hernie neben der Verdrängung des Herzens nach rechts den oben erwähnten, weit in den Thoraxraum vom Mediastinum zur Brustwand ausgespannten Bogen, über dem sich durch typische Lungenzeichnung charakterisiertes Lungengewebe befindet, unter dem man luftgefüllte Eingeweideteile 'unterscheiden kann. Die Schwierigkeit besteht nun darin, daß zu entscheiden ist, ob dieser Bogen durch das Zwerchfell + oberer Begrenzung der Magenblase gebildet wird - es wäre dann eine $R$. $d$. sichergestellt - oder ob der Bogen die obere Magenblasenbegrenzung allein darstellt, was nur bei einem Loche im Zwerchfell, also bei der Hernie, der Fall sein kann. Die Zahlen der für diese Differenzierung angegebenen methodologischen Feinheiten stehen wie so häufig in umgekehrtem Verhältnis zur restlosen Sicherstellung der Diagnose. Es ist, wie es ja bei der bisherigen Kleinheit des Materials erklärlich ist, nur ein Tasten nach der endgültigen Sicherheit der Erkennung zu konstatieren. Diese Unsicherheit illustriert treffend die Gegenüberstellung Beckers, die dartut, daß von den als $R$. d. angesprochenen Fällen über die Hälfte von verschiedenen Autoren verschieden beurteilt wurden. Hier ist auch der bekannte Fall zu nermen, der 1890 von Stinzing als angeborene Dextrokardie bezeichnet wurde, der späterhin so häufig geröntgt wurde, daß eine Hautverbrennung die Folge war. Die zahlreichen Untersucher standen sich in zwei Lagern, hier $\mathrm{R}$. d. hier Hernia diaphragmatica, gegenuber, bis schließlich die Sektion im Jahre 1910 der hauptsächlich von Hess und Hildebrandt verfochtenen Ansicht, daß eine R. d. vorliege, recht gab.

In einem anderen Falle glaubte Lotze nach genauerer Untersuchung und nach kritischer Würdigung seiner Befunde mit Sicherheit eine R. d. annehmen zu können, doch deckte dif Sektion (Riesel) ein Jahr später eine Zwerchfellhernie auf.

Wir können aus diesen diagnostischen Schwierigkeiten erkennen, da $B$ bisher letzten Endes nur die Autopcie Klarheit geben kann. Unsere Beobachtung ist deshalb sehr wertvoll, weil wir unsere klinische Diagnose durch die Sektion kontrollieren konnten. Die Schwere des Falles erlaubte, wie schon bemerkt, leider keine weiteren Untersuchungen, die zur Kontrolle der angegebenen Methoden hätte dienen können; dagegen war einwandfre das bereits von vielen Autoren als für die R. d. typische Phänomen die geringe, ater dem*' gesunden Zwerchfell gleichmäßige $\mathrm{Be}-$ weglichkeit des $\mathrm{zu}$ differenzierenden Bogens, vorhanden, nicht dagegen eine paradoxe Verschieblichkeit, die ein sirheres Charakteristikum der Hernie ist. Außerdem zeigte der oben genannte Grenzbogen in verschiedenen Aufnahmen immer eine Konstanz seiner Form. Nach unserer Beobachtung können demnach die gleichsinnige Bewegung des Grenzbogens sowie die Konstanz des Befundes in mehreren Aufnahmen als sichere Posten in der röntgenølogischen Differentialdiagnose zwischen Hernie und R. d. zugunsten der letzteren gebucht werden. Ich möchte noch erwähnen, daß in einigen Fällen (Beltz, Arnsperger, Baetge, Frank k) durch den Nachweis zweier getrennter war. Doch ist dieser eindeutige Befund leider nur als Ausnahme $\mathrm{zu}$ bezeichnen.

Einzelne Autoren suchten auf dem Wege über den Phrenikus zum Ziele zu kommen, indem sie die von $\mathrm{Jamin}^{1}$ ) angegebene elektrische Reizung des Nerven vor dem Röntgenschirm zur Kontraktion und damit zur Sichtbarmachung des Zwerchfells benutzen wollten. Wenn dieses Phănomen vorhanden sei, solle 
es für eine R. d. gegen eine Hernie sprechen, Nun ist aber die nervöse Zwerchfellreizung einerseits abhängig von einem intakten, reizleitenden Nerven, anderseits von dem Vorhandensein noch genügend kontraktionsfähiger Muskelbündel im Zwerchfell. Da aber beide Voraussetzungen bei der R. d. wohl in den meisten Fällen fehlen, so ist meines Erachtens diese Methode für eine Differenzierung wertlos.

Weit aussichtsreicher scheint die in neuester Zeit von Goetze $^{1}$ ) angegebene Röntgenuntersuchung der Bauchhöhle bei Sauerstoffullung zu sein. Goetze konnte dabei das Zwerchfell deutlich zur Darstellung bringen und sogar geringe Veränderungen, wie Metastasen, nachweisen. Es ist einleuchtend, da $B$ diese Methode, eventuell in Kombination mit der Kontrastfüllung des Magens und Dickdarms. berufen sein kann, das differentialdiagnostische Halbdunkel zwischen R. d. und Hernie zu erleuchten.

Die Differenzierung hat praktisches Interesse insofern, als bei der sicheren Erkennung einer R. d. alle ungünstigen therapeutischen Eingriffe, Punktionen und Operationen vermieden werden können, wie es bisher des öfteren vorgekommen ist.

(SchluB folgt.) 